\title{
Time-Frequency Analysis of the Surface Electrocardiogram in Atrial Fibrillation for Monitoring and Predicting Antiarrhythmic Drug Effects
}

\author{
D Husser ${ }^{1}$, M Stridh ${ }^{2}$, L Sornmo ${ }^{2}$, SB Olsson ${ }^{3}$, HU Klein ${ }^{1}$, A Bollmann ${ }^{1,3}$ \\ ${ }^{1}$ Department of Cardiology, Otto-von-Guericke-University Magdeburg, Germany \\ ${ }^{2}$ Department of Electroscience, Lund University, Sweden \\ ${ }^{3}$ Department of Cardiology, Lund University, Sweden
}

\begin{abstract}
This study explored the potential clinical value of Time-Frequency Analysis (TFA) of the surface ECG for monitoring and predicting atrial drug action in atrial fibrillation $(A F)$.

Baseline two-minute ECG recordings were made in 42 patients with persistent $A F$ and after oral flecainide $(n=24)$ or amiodarone $(n=18)$ initiation. After spatiotemporal QRST cancellation, TFA was performed and (1) mean fibrillatory rate, (2) rate stability, and (3) exponential decay of fundamental and harmonic frequency components were determined.

Patients in whom AF terminated $(n=12)$ had a lower atrial rate and a smaller exponential decay at baseline than patients who remained in $A F$. In the remaining 30 patients, drug loading organized $A F$ as expressed by (1) decrease of atrial rate, (2) increase of ECG segments with stable $A F$, and (3) decrease of exponential decay.

In conclusion, TFA has the potential to predict and monitor individual antiarrhythmic drug responses in $A F$.
\end{abstract}

\section{Introduction}

Prompted by the likely usefulness of a non-invasive technique that assesses the average fibrillatory rate from the surface electrocardiogram (ECG) for exploration of atrial fibrillation (AF) pathophysiology, frequency analysis of AF was developed. The dominant atrial fibrillatory rate has been shown to be of potential clinical value for monitoring and predicting antiarrhythmic drug responses as well as for predicting atrial defibrillation thresholds and AF recurrence following cardioversion (for a review see [1]).

More recently, Time-Frequency Analysis has been developed allowing more detailed charaterization of the fibrillatory process.[1-3] Potential clinical applications of this new method have, however, not been explored.

The purpose of this study was, therefore, to assess the ability of novel ECG parameters to monitor and predict antiarrhythmic drug actions in patients with AF.

\section{Methods}

\subsection{Patients}

This observational study included 44 consecutive patients with $\mathrm{AF}>24$ hours duration (range 24 hours to 96 months). Baseline ECG recordings were not suitable for further analysis in two patients which were excluded. The study population that comprised the remaining 42 patients is characterized in Table 1. All patients provided informed consent for study participation.

Table 1. Characteristics of the study population.

\begin{tabular}{lc}
\hline Age, years & $62 \pm 12$ \\
Male/Female & $25 / 17$ \\
AF duration, months & $17 \pm 25$ \\
Heart disease & \\
$\quad$ None (lone AF) & 10 \\
Systemic hypertension & 20 \\
Coronary artery disease & 3 \\
Dilated cardiomyopathy & 3 \\
Valvular heart disease & 6 \\
LAD, mm & $45 \pm 5$ \\
LVEF, \% & $54 \pm 14$ \\
Rate control drugs * & 19 \\
Digitalis & 15 \\
Beta blocker & 15 \\
Calcium channel blocker & \\
* more than one possible, LAD = left atrial diameter, \\
LVEF = left ventricular ejection fraction
\end{tabular}

\subsection{ECG acquisition and analysis}

Before antiarrhythmic drug initiation and after oral antiarrhythmic drug loading (see below for details), a two-minute ECG (Predictor, Dr. Kaiser Medizintechnik $\mathrm{GmbH}$, Bad Hersfeld, Germany) was recorded in all patients with all subjects relaxed in a supine position after a five-minute equilibration period. 
Time-Frequency Analysis was performed on bipolar, low $\mathrm{C} 1$ lead (below left clavicle to right sternal border at the $6^{\text {th }}$ intercostal space, "bipolar modification of V1". [4]
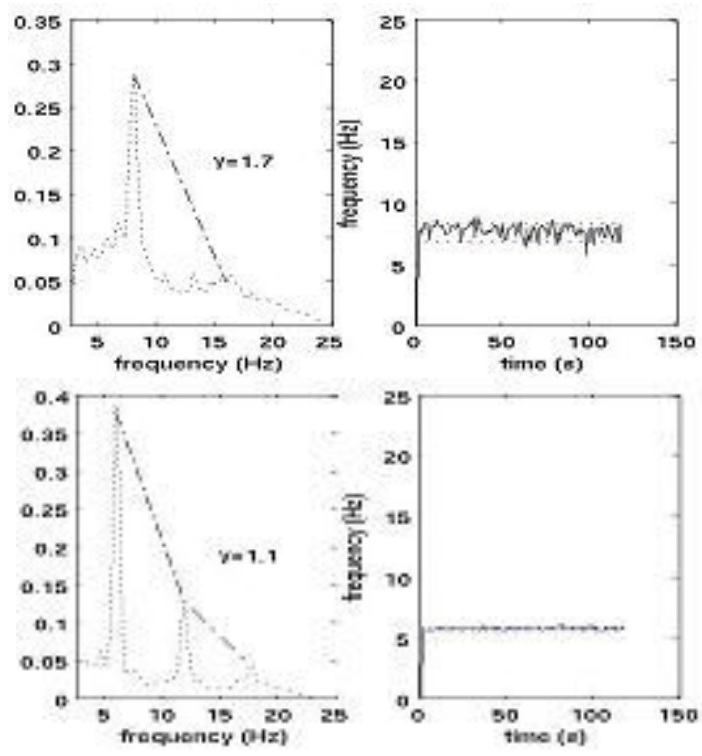

Figure 1. Time-Frequency Analysis after QRST cancellation (not shown) at baseline (top panels) and after flecainide intake (bottom panels). Frequency power spectrum from which the exponential decay $(\gamma)$ was calculated (left boxes). Instantaneous fibrillatory rate over the two-minute recording from which mean fibrillatory rate and rate stability were obtained (right boxes). Please note the more pronounced dominant and harmonic peaks after drug administration which is expressed by a decreased exponential decay. Drug administration is also associated with atrial rate reduction and rate stabilization.

Time-Frequency Analysis has been described previously in detail.[1-3] Briefly, after analog-to-digital conversion $(2000 \mathrm{~Hz}, 12$ bit, $0.05-300 \mathrm{~Hz}$ ) electrograms were stored on optical disk and transferred to a personal computer. After high-pass filtering to remove baseline wander, QRST complexes were subtracted using spatiotemporal QRST cancellation. Since the dominant frequency component of interest is within the $4-9 \mathrm{~Hz}$ range, the resulting fibrillatory baseline signal was downsampled to $50 \mathrm{~Hz}$ and subjected to spectral analysis. The time-frequency distribution of the atrial signal (obtained by short-term Fourier transform) is decomposed such that each spectrum can be modeled as a frequencyshifted and amplitude-scaled version of the spectral profile. This procedure is based on a spectral profile, dynamically updated from previous spectra, which is matched to each new spectrum using weighted least squares estimation. The frequency shift needed to achieve optimal matching then yields a measure of instantaneous fibrillatory rate of a 2.5-second ECG segment (overlapping with one segment each second) and is trended as a function of time.

Frequencies were converted to fibrillatory rates with its unit fibrillations per minute (fpm) as advocated previously (rate $=$ frequency $* 60$ ).[1] The following parameters were determined: (1) mean fibrillatory rate (in fpm) defined as average of instantaneous fibrillatory rates over the two-minute ECG segment, (2) stability of instantaneous, second-to-second fibrillatory rate (in \%) defined as the ECG segment proportion with consecutive rate differences $<6 \mathrm{fpm}$, and (3) exponential decay defined as decay of the curve that connects power maxima of dominant and harmonic frequency components in the $4-25 \mathrm{~Hz}$ frequency band (Figure 1).

The relation between dominant and harmonic frequency components has recently been suggested by others [5] to represent a marker of "global AF organization". Higher harmonic frequency components are consequently associated with a smaller exponential decay and indicate more organized rhythms.[2,3]

\subsection{Study protocol}

Antiarrhythmic drugs were initiated on an inpatient basis under continuous electrocardiographic monitoring using standard doses (flecainide $200-400 \mathrm{mg} /$ day, $\mathrm{n}=24$; amiodarone $1200 \mathrm{mg} /$ day for 7 days, $\mathrm{n}=18$ ). The specific medication depended on the referring physician's preference and on concomitant heart disease. All patients were adequately anticoagulated using either oral anticoagulation therapy with an international normalized ratio between 2.5 and 3.5 or weight-adapted low molecular weight heparin following exclusion of left atrial thrombus formation by transesophageal echocardiography.

\subsection{Statistical analysis}

All continuous variables are presented as mean \pm one standard deviation. ECG parameters before and after drug administration were compared using Student's $t$-test for paired data. Clinical variables and ECG parameters were compared between patients who converted to sinus rhythm within 48 hours after drug initiation to those who did not convert. Differences between groups were assessed using Student's $t$-test for unpaired data or MannWhitney $U$ test for continuous variables and chi-square test for categoric variables. Independent predictors for AF termination were evaluated using multivariate regression analysis. and receiver operating characteristic curves were constructed and the area under the curve (AUC) was calculated. Associations among variables were explored using Pearson or Spearman correlation. A p value $<.05$ was considered statistically significant. 


\section{Results}

Baseline ECG characteristics are summarized below.

Table 2. Baseline ECG characteristics.

\begin{tabular}{lcc}
\hline \multicolumn{1}{c}{ Parameter } & Mean \pm one SD & Range \\
\hline Mean fibrillatory rate, fpm & $402 \pm 41$ & $313-541$ \\
Rate stability, \% & $13 \pm 4$ & $4-28$ \\
Exponential decay & $1.24 \pm 0.24$ & $0.73-1.70$ \\
\hline
\end{tabular}

Exponential decay correlated with mean fibrillatory rate $(\mathrm{R}=.761, \mathrm{p}<.001, \mathrm{R}=0.759, \mathrm{p}<.001)$ and rate stability $(\mathrm{R}=-.427, \mathrm{p}=.005, \mathrm{R}=-.545, \mathrm{p}=.003)$ at baseline and after drug loading as well as with $\mathrm{AF}$ duration $(\mathrm{R}=.355$, $\mathrm{p}=.025)$. Patients with AF duration $<1$ month $(\mathrm{n}=5)$ had a lower mean fibrillatory rate $(361 \pm 40$ vs. $406 \pm 38 \mathrm{fpm}$, $\mathrm{p}=.019)$ and a smaller exponential decay $(1.0 \pm 0.1$ vs. $1.3 \pm 0.2, \mathrm{p}=.039)$ than patients with longer arrhythmia duration $(n=37)$. The use of verapamil-type calcium channel blockers was associated with a lower fibrillatory rate $(385 \pm 35$ vs. $411 \pm 41 \mathrm{fpm}, \mathrm{p}=.046)$. There was no other relationship between any clinical or echocardiographic and ECG variable.

Drug loading restored sinus rhythm in 12 patients, while AF was persistent in the others. Characteristics of patients with or without AF termination are compared below.

Table 3. Characteristics of patients with or without AF termination.

\begin{tabular}{lccc}
\hline \multicolumn{4}{c}{ AF termination } \\
\hline & yes $(\mathrm{n}=12)$ & no $(\mathrm{n}=30)$ & $\mathrm{p}$ \\
\hline Age, years & $63 \pm 14$ & $62 \pm 11$ & $\mathrm{NS}$ \\
Male & 6 & 19 & $\mathrm{NS}$ \\
Female & 6 & 11 & \\
AF duration, months & $4 \pm 5$ & $21 \pm 28$ & .013 \\
Heart disease & 6 & 26 & .012 \\
Lone AF & 6 & 4 & \\
LAD, mm & $41 \pm 4$ & $47 \pm 4$ & .001 \\
LVEF, \% & $60 \pm 6$ & $52 \pm 16$ & $\mathrm{NS}$ \\
Flecainide & 11 & 13 & .004 \\
Amiodarone & 1 & 17 & \\
Digitalis & 3 & 16 & $\mathrm{NS}$ \\
Beta blocker & 4 & 11 & $\mathrm{NS}$ \\
Calcium blocker & 7 & 8 & .053 \\
Fibrillatory rate, fpm & $375 \pm 37$ & $412 \pm 38$ & .006 \\
Rate stability, $\%$ & $14 \pm 6$ & $12 \pm 3$ & $\mathrm{NS}$ \\
Exponential decay & $1.13 \pm 0.19$ & $1.28 \pm 0.25$ & .034 \\
\hline
\end{tabular}

Multivariate regression analysis revealed left atrial diameter $(\mathrm{Beta}=.-634, \mathrm{p}=.024)$ and atrial rate $(\mathrm{Beta}=-.073$, $\mathrm{p}=.045$ ) to be the only independent predictors for $\mathrm{AF}$ termination.

Drug loading (1) decreased mean fibrillatory rate
$(400 \pm 77$ vs. $322 \pm 52 \mathrm{fpm})$, (2) increased rate stability $(12 \pm 3$ vs. $31 \pm 22 \%)$, and (3) decreased exponential decay $(1.3 \pm 0.2$ vs. $0.9 \pm 0.3, \mathrm{p}<.001$ for all variables) and also decreased ventricular rate $(90 \pm 18$ vs. $81 \pm 17, \mathrm{p}=.005)$. Both fibrillatory rate decrease and rate stability increase were more pronounced with flecainide than with amiodarone (Figure 2). There was no relationship between baseline ECG parameters and the degree of their changes.

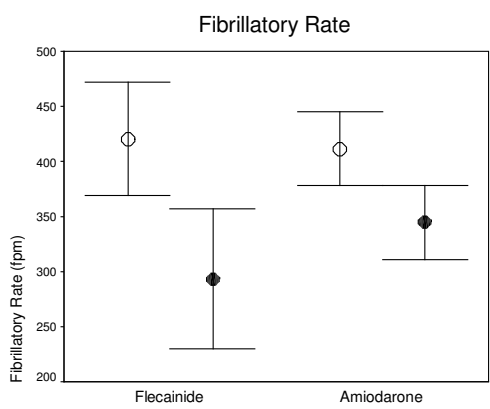

Baseline vs. Drug $\mathrm{p}<.001$ for Flec $\mathrm{p}<.001$ for Amio

Change Flecainide vs. Amiodarone $\mathrm{p}=.015$

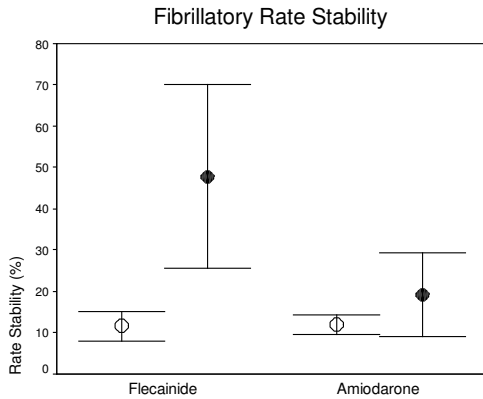

Baseline vs. Drug

$\mathrm{p}=.001$ for Flec

$\mathrm{p}=.011$ for Amio

Change Flecainide vs. Amiodarone $\mathrm{p}=.002$

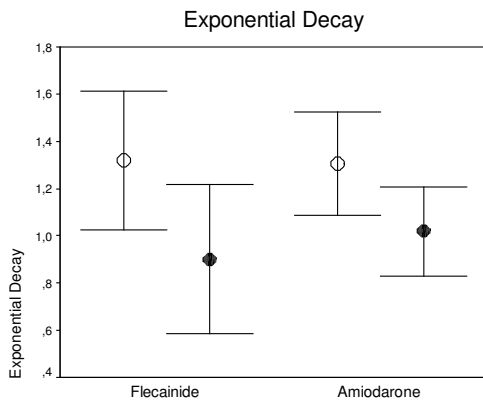

Baseline vs. Drug $\mathrm{p}=.001$ for Flec $\mathrm{p}=.001$ for Amio

Change Flecainide vs. Amiodarone $\mathrm{p}=.272$

Figure 2. Influence of oral flecainide or amiodarone on ECG parameters $(0=$ baseline, $\bullet=$ drug $)$.

\section{Discussion and conclusions}

This study, for the first time, applied novel ECG signal processing techniques allowing determination of fibrillatory rate, rate stability and exponential decay in the clinical setting. It was clearly demonstrated that these Time-Frequency Analysis parameters changed with the 
administration of flecainide or amiodarone and were related with $\mathrm{AF}$ termination following drug initiation.

\subsection{Monitoring and predicting atrial drug effects during $\mathrm{AF}$}

Class I and III antiarrhythmic drugs have been shown to increase atrial cycle length (decrease fibrillatory rate) which coincides with increased refractoriness and decreased conduction velocity.[6] These mixed effects might explain why atrial cycle length is closely related with baseline but not with refractoriness after antiarrhythmic drug administration.[7] Even though the individual contribution of refractoriness prolongation or conduction slowing might currently not be differentiated, atrial drug action may be explored by fibrillatory rate monitoring from the surface ECG. Indeed, a substantial rate reduction following intravenous sotalol, ibutilide, cibenzoline or procainamide as well as oral flecainide, amiodarone, bepidril or verapamil (for a review see [8]) has been observed previously and in this study.

This study extends previous work by taking atrial rate stability and exponential decay into account. While rate stability clearly attempts to quantifiy the temporal phenomenon of changing rates inherent to $\mathrm{AF}$, the exponential decay exhibits a close relation with a socalled "organization index". This index has been previously introduced by others [5] as measure for "global AF organization" from intracardiac recordings. Besides the aforementioned effects on atrial refractoriness and conduction, antiarrhythmic drugs have been shown to decrease dispersion of atrial refractoriness [7] which spatially organizes the fibrillatory process. In fact, rate stability increased and exponential decay decreased after antiarrhythmic drug administration.

A low fibrillatory rate in association with high rate stability and low decay, which were all interrelated in our study, reflect a more organized AF with fewer wavefronts, while a higher fibrillatory rate with low rate stability and higher decay is more likely found in disorganized AF with more wavefronts. This notion is supported by previous mapping studies showing a close relation between rate and complexity of fibrillatory activation [9] and our finding that patients with shorter AF duration had a lower fibrillatory rate and a smaller exponential decay. Under the latter conditions (fewer wavelets), the statistical likelihood that all wavelets might extinguish simultaneously and terminate the fibrillatory process after drug administration is higher than in patients with higher fibrillatory rate and a larger exponential decay (more wavelets).

Although not being the main purpose of this study, comparison of flecainide's and amiodarone's effects revealed a greater rate reduction with flecainde which is consistent with previous investigations using intracardiac recordings [6,7] and may be partly attributed to more pronounced conduction slowing with class IC drugs [6] but also to different drug onset dynamics.

\subsection{Conclusion}

Time-Frequency Analysis has the potential to monitor and predict individual antiarrhythmic drug responses in AF patients.

\section{References}

[1] Bollmann A, Husser D, Stridh M, et al. Frequency measures obtained from the surface electrocardiogram in atrial fibrillation research and clinical decision-making. $\mathrm{J}$ Cardiovasc Electrophysiol. 2003;14:S154-S161.

[2] Stridh M, Sornmo L, Meurling CJ, Olsson SB. Characterization of atrial fibrillation using the surface ECG: time-dependent spectral properties. IEEE Trans Biomed Eng. 2001;48:19-27.

[3] Stridh M, Sornmo L, Meurling CJ, Olsson SB. Sequential characterization of atrial tachyarrhythmias based on ECG time-frequency analysis. IEEE Trans Biomed Eng. 2004;51:100-14.

[4] Waktare JE, Gallagher MM, Murtagh AM, Camm AJ, Malik M. Optimum lead positioning for recording bipolar atrial electrocardiograms during sinus rhythm and atrial fibrillation. Clin Cardiol. 1998;21:825-30

[5] Everett TH, 4th, Moorman JR, Kok LC, Akar JG, Haines DE. Assessment of global atrial fibrillation organization to optimize timing of atrial defibrillation. Circulation. 2001;103:2857-61.

[6] Wijffels MC, Dorland R, Mast F, Allessie MA. Widening of the excitable gap during pharmacological cardioversion of atrial fibrillation in the goat: effects of cibenzoline, hydroquinidine, flecainide, and d-sotalol. Circulation. 2000;102:260-7

[7] Fynn SP, Todd DM, Hobbs WJC, et al. Effect of amiodarone on dispersion of atrial refractoriness and cycle length in patients with atrial fibrillation. J Cardiovasc Electrophysiol. 2003;14:485-91.

[8] Husser D, Stridh M, Sornmo L, et al. Analysis of the surface electrocardiogram for monitoring and predicting antiarrhythmic drug effects in atrial fibrillation. Cardiovasc Drugs Ther. in press.

[9] Konings KT, Kirchhof CJ, Smeets JR, et al. High-density mapping of electrically induced atrial fibrillation in humans. Circulation. 1994;89:1665-80.

Address for correspondence

Andreas Bollmann, MD, FESC

Department of Cardiology

Good Samaritan Hospital

1225 Wilshire Blvd.

Los Angeles, CA 90017

USA

Email: andreas.bollmann@kard.lu.se or

andreas.bollmann@medizin.uni-magdeburg.de 